\title{
Desafios e paradigmas para o exercício da preceptoria frente ao feedback formativo em residências de Enfermagem
}

\author{
Challenges and paradigms for the exercise of preceptorship in face of formative feedback in
} Nursing residencies

Retos y paradigmas para el ejercicio de la preceptoría ante la retroalimentación formativa en las residencias de Enfermeira

Carlos Antonio Farias Sales Junior

ORCID: https://orcid.org/0000-0002-9700-9305 Universidade do Estado do Pará, Brasil E-mail: junior_sales1@hotmail.com

Ilma Pastana Ferreira

ORCID: https://orcid.org/0000-0002-9152-3872 Universidade do Estado do Pará, Brasil E-mail: ilma.pastana@uepa.com

Ruhan da Conceição Sacramento ORCID: https://orcid.org/0000-0003-3629-7945 Universidade do Estado do Pará, Brasil E-mail: ruhan.sacramrnto@gmail.com

Marcella Lacerda Moura

ORCID: https://orcid.org/0000-0002-7831-2317 Universidade do Estado do Pará, Brasil E-mail: marcella_lmoura@gmail.com

Vera Regina da Cunha Menezes Palacios

ORCID: https://orcid.org/0000-0003-0300-7972 Universidade do Estado do Pará, Brasil E-mail: verareginapalacios@gmail.com

Márcia Bitar Portella

ORCID: https://orcid.org/0000-0002-4602-7618 Universidade do Estado do Pará, Brasil E-mail: marciabitar@gmail.com

Larissa Cristina Machado de Barros ORCID: https://orcid.org/0000-0002-2836-7707 Universidade do Estado do Pará, Brasil E-mail: laridebarros@gmail.com

\begin{abstract}
Resumo
Objetivo: identificar, por meio da busca na literatura, as evidências que cerceiam a importância do feedback, bem como os desafios e paradigmas para o exercício da preceptoria e do feedback formativo no contexto das residências em enfermagem. Método: Pesquisa de abordagem qualitativa, com natureza descritiva, da tipologia Revisão Integrativa da Literatura. A coleta de dados ocorreu a partir dos seguintes critérios de inclusão: Textos com resumos e disponíveis de forma completa online; artigos nos idiomas português e inglês; estudos publicados de 2016 a 2021 . A questão que norteou a busca foi: "Quais as evidências nas bases de dados sobre o exercício da preceptoria frente ao feedback formativo em residências de enfermagem?". As bases de dados utilizadas foram Literatura LatinoAmericana e do Caribe em Ciências da Saúde e Medical Literature Analysis and Retrieval System Online e BDENF Enfermagem, por meio do Portal da Biblioteca Virtual em Saúde com os Descritores - descritores controlados Feedback formativo; Enfermagem; Internato não médico; Preceptoria; Educação em Enfermagem; Educação de pósgraduação em enfermagem; e com as palavras-chave - descritores não controlados: Pós-graduação; Feedback e Preceptor. Resultados: Foram selecionados 7 artigos, os quais foram analisados e originaram 3 categorias. 1) A importância do Feedback Formativo na formação em residências; 2) O papel do preceptor Enfermeiro perante os residentes e ao Feedback Formativo e 3) Entraves para a atuação na preceptoria em residências e o processo de Feedback Formativo. Considerações Finais: A partir dessa revisão de literatura, foi possível identificar a importância do feedback formativo, bem como da preceptoria na ambiência das residências de enfermagem.
\end{abstract}

Palavras-chave: Feedback formativo; Enfermagem; Internato não médico; Preceptoria; Educação em Enfermagem. 


\begin{abstract}
Objective: to identify, through a literature search, the evidence surrounding the importance of feedback, as well as the challenges and paradigms for the exercise of preceptorship and formative feedback in the context of nursing residencies. Method: Research with a qualitative approach, with a descriptive nature, of the Integrative Literature Review typology. Data collection took place based on the following inclusion criteria: Texts with abstracts and available completely online; articles in Portuguese and English; studies published from 2016 to 2021. The question that guided the search was: "What is the evidence in the databases on the exercise of preceptorship in face of formative feedback in nursing homes?". The databases used were Latin American and Caribbean Literature in Health Sciences and Medical Literature Analysis and Retrieval System Online and BDENF - Nursing, through the Virtual Health Library Portal with Descriptors - controlled descriptors - formative feedback; Nursing; Non-medical boarding school; Preceptorship; Nursing Education; Graduate nursing education; and with the keywords - uncontrolled descriptors: Graduate; Feedback and Preceptor. Results: 7 articles were selected, which were analyzed and originated 3 categories. 1) The importance of Formative Feedback in training in residencies; 2) The role of the Nurse preceptor with the residents and with the Formative Feedback and 3) Barriers for the performance of preceptorship in homes and the Formative Feedback process. Final Considerations: From this literature review, it was possible to identify the importance of formative feedback, as well as preceptorship in the environment of nursing homes.
\end{abstract}

Keywords: Formative feedback; Nursing; Non-medical boarding school; Preceptorship; Nursing Education.

\title{
Resumen
}

Objetivo: identificar, mediante una búsqueda bibliográfica, la evidencia en torno a la importancia de la retroalimentación, así como los desafíos y paradigmas para el ejercicio de la preceptoría y la retroalimentación formativa en el contexto de las residencias de enfermería. Método: Investigación con enfoque cualitativo, de carácter descriptivo, de la tipología Revista Integrativa de Literatura. La recolección de datos se realizó con base en los siguientes criterios de inclusión: Textos con resúmenes y disponibles completamente en línea; artículos en portugués e inglés; estudios publicados de 2016 a 2021. La pregunta que orientó la búsqueda fue: "¿Cuál es la evidencia en las bases de datos sobre el ejercicio de la preceptoría frente a la retroalimentación formativa en los hogares de ancianos?". Las bases de datos utilizadas fueron Literatura Latinoamericana y del Caribe en Ciencias de la Salud y Sistema de Análisis y Recuperación de Literatura Médica en Línea y BDENF - Enfermería, a través del Portal de la Biblioteca Virtual en Salud con Descriptores - descriptores controlados - retroalimentación formativa; Enfermería; Internado no médico; Tutela; Educación en enfermería; Educación de posgrado en enfermería; y con las palabras clave descriptores no controlados: Graduado; Retroalimentación y Preceptor. Resultados: Se seleccionaron 7 artículos, los cuales fueron analizados y originaron 3 categorías. 1) La importancia de la Retroalimentación Formativa en la formación en residencias; 2) El rol del Enfermero preceptor con los residentes y con la Retroalimentación Formativa y 3) Barreras para el desempeño de la preceptoría en los hogares y el proceso de Retroalimentación Formativa. Consideraciones finales: A partir de esta revisión de la literatura, fue posible identificar la importancia de la retroalimentación formativa, así como la preceptoría en el entorno de los hogares de ancianos.

Palabras clave: Retroalimentación formativa; Enfermería; Internado no médico; Tutela; Educación en enfermería.

\section{Introdução}

Os programas de residência em enfermagem tiveram seu marco de origem nos Estados Unidos da América, servindo de modelo e se perpetuando internacionalmente. No cenário brasileiro, a residência em enfermagem tem seu pontapé inicial em São Paulo no ano de 1961, no Hospital Infantil do Morumbi. Desde então iniciou-se a propagação dessa vertente de especialização no Brasil. No entanto, apenas no ano de 1996 o Projeto de Lei no 2.264, instituiu e regulamentou a Residência em Enfermagem na modalidade de Pós-graduação (Brasil, 1996; Martins et al., 2016; Fajardo et al., 2010; Freitas, Sé, Gonçalves \& Pereira, 2021).

Atualmente, as residências em Enfermagem estão inseridas na Residência em Área Profissional da Saúde, respaldada pela Lei $\mathrm{n}^{\circ}$ 11.129, de 30 de junho de 2005, que dispõe acerca da Residência Multiprofissional e em Área Profissional da Saúde. Esta lei constitui esse modelo de formação em saúde, o qual alcança seus objetivos por meio de ações interministeriais entre os Ministérios da Educação e o Ministério da Saúde, os quais criam e viabilizam estratégias para culminar na formação de profissionais comprometidos com o Sistema Único de Saúde (Santos et al., 2017).

As residências em saúde se caracterizam como modelos formativos que propulsionam o ensino-aprendizagem de forma experiencial pautadas na reflexão crítica sobre situações cotidianas assistenciais. As residências são constituídas em 
80\% de carga horaria prática e 20\% carga horaria teórica (Santos et al., 2017; Brasil, 2014).

Nesse contexto, os residentes de enfermagem necessitam de profissionais estimulando esse processo. Assim, a preceptoria surge a fim de mediar o ensino-aprendizagem. Nesse sentido, para os residentes de enfermagem, a preceptoria deve ser realizada por enfermeiros inseridos nos seus próprios âmbitos de trabalho, os quais necessitam criar mecanismos de execução das atividades no cotidiano assistencial juntamente aos residentes (Ferreira, Dantas \& Valente, 2018).

O Preceptor é responsável pela supervisão, orientação e avaliação do residente no campo de prática. Como um dos mecanismos de avaliação destaca-se o Feedback formativo, o qual visa fornecer subsídios para que o residente identifique as lacunas do conhecimento, podendo assim autorregular sua aprendizagem (Santos et al., 2017; Borges, 2014).

O feedback formativo é uma das influências mais poderosas na aprendizagem e no desempenho, uma vez que possibilita ao residente a oportunidade para se beneficiar da crítica, do raciocínio, do conselho e do apoio de outro profissional, nesse caso, o preceptor (Johnson et al., 2019).

No entanto, nota-se que a preceptoria aliada ao feedback formativo apresenta diversos desafios. Dessa forma, busca-se uma visão ampliada acerca desses processos atrelados. Assim, os objetivos dessa pesquisa foram identificar, por meio da busca na literatura, as evidências que cerceiam a importância do feedback, bem como os desafios e paradigmas para o exercício da preceptoria e do feedback formativo no contexto das residências em enfermagem.

\section{Metodologia}

Pesquisa de abordagem qualitativa, com natureza descritiva, da tipologia Revisão Integrativa da Literatura (RIL). A RIL é considerada um mecanismo de investigação, o qual possibilita o usufruto de pesquisas experimentais e não experimentais, sendo realizadas sínteses vislumbrando a compreensão de um fenômeno em análise elencado a partir de uma lacuna encontrada (Sousa, 2017).

Dessa forma, a coleta de dados ocorreu no período de abril a maio de 2021, a partir dos seguintes critérios de inclusão: Textos com resumos e disponíveis de forma completa online; artigos nos idiomas português e inglês; estudos publicados de 2016 a 2021. Portanto, excluíram-se: artigos duplicados entre as bases; artigos que não possuíam relação com o tema em estudo e/ou que não respondessem as questões norteadoras; revisões integrativas e sistemáticas da literatura; monografias; dissertações de mestrado e teses de doutorado.

Nesse sentido, a questão que norteou a busca foi: "Quais as evidências nas bases de dados sobre o exercício da preceptoria frente ao feedback formativo em residências de enfermagem?".

As bases de dados utilizadas foram Literatura Latino-Americana e do Caribe em Ciências da Saúde (LILACS) e Medical Literature Analysis and Retrieval System Online (MEDLINE) e BDENF - Enfermagem, por meio do Portal da Biblioteca Virtual em Saúde (BVS) do Ministério da Saúde com os Descritores - descritores controlados - Feedback formativo; Enfermagem; Internato não médico; Preceptoria; Educação em Enfermagem; Educação de pós-graduação em enfermagem; e com as palavras-chave - descritores não controlados: Pós-graduação; Feedback e Preceptor. Foram realizadas as seguintes combinações entre o descritores e palavras-chave com o operador booleano AND: "Preceptoria AND Feedback Formativo AND Enfermagem"; "Formative feedback AND Nursing"; "Enfermagem AND Internato não médico AND Educação em enfermagem AND Feedback"; "Enfermagem AND Preceptor AND Pós-graduação AND Feedback" e "Educação de pós-graduação em enfermagem AND Enfermagem AND Preceptor”. Nas bases de dados, estratégias de busca avançada foram utilizadas como busca por título, resumo, tema e uso de critérios de inclusão e exclusão para obter o número de artigos selecionados para amostragem final.

Diante da análise e leitura de forma integral dos artigos selecionados, os estudos a compor a amostra final 
permaneceram em detrimento de responderem as questões norteadoras, bem como objetivos da presente pesquisa. Frisa-se que para a realização da extração de dados pertinentes dos artigos selecionados, fez-se usufruto de um formulário de coleta de dados, o qual foi idealizado pelo autor para esse fim. O banco de dados continha informações sobre a identificação dos estudos e acerca de seus conteúdos.

Assim, após a seleção da amostra final, os estudos selecionados foram analisados quantos aos principais aspectos referentes a importância do processo de feedback formativo para residentes de enfermagem, assim como sobre o papel do preceptor enfermeiro frente a essa ferramenta e sua função de preceptoria. No que tange a etapa referente a análise dos dados utilizou-se o mecanismo de análise de conteúdo proposta por Bardin, a qual consiste em: 1) Pré-análise; 2) Exploração do material; e 3) Tratamento dos resultados: inferência e interpretação (Bardin, 2016), possibilitando a categorização dos artigos componentes da amostra final. Dessa forma, os artigos foram discutidos de maneira sistemática, vislumbrando um melhor entendimento no que concerne à temática. Elaborou-se a síntese final de forma descritiva, condizendo aos resultados, discussões e às conclusões obtidos em cada artigo.

\section{Resultados e Discussão}

As combinações entre descritores e palavras-chave na BVS, revelam um quantitativo de 283 estudos encontrados, os quais foram analisados conforme critérios de inclusão e exclusão pré-estabelecidos de modo a realizar uma busca sistemática acerca das questões norteadoras e critérios de elegibilidade de artigos. Assim, após aplicação de critérios primários, 270 estudos foram excluídos, resultando na pré-seleção de 13 artigos a serem lidos integralmente a fim da identificação de itens, os quais contemplassem o objeto deste estudo. Dessa forma, apenas 8 artigos foram selecionados a compor a amostra final do levantamento bibliográfico, conforme Fluxograma 1.

Fluxograma 1 - Seleção dos Estudos que abordam a importância do feedback formativo e o papel do preceptor enfermeiro em residências, no período de 2016 a 2021.

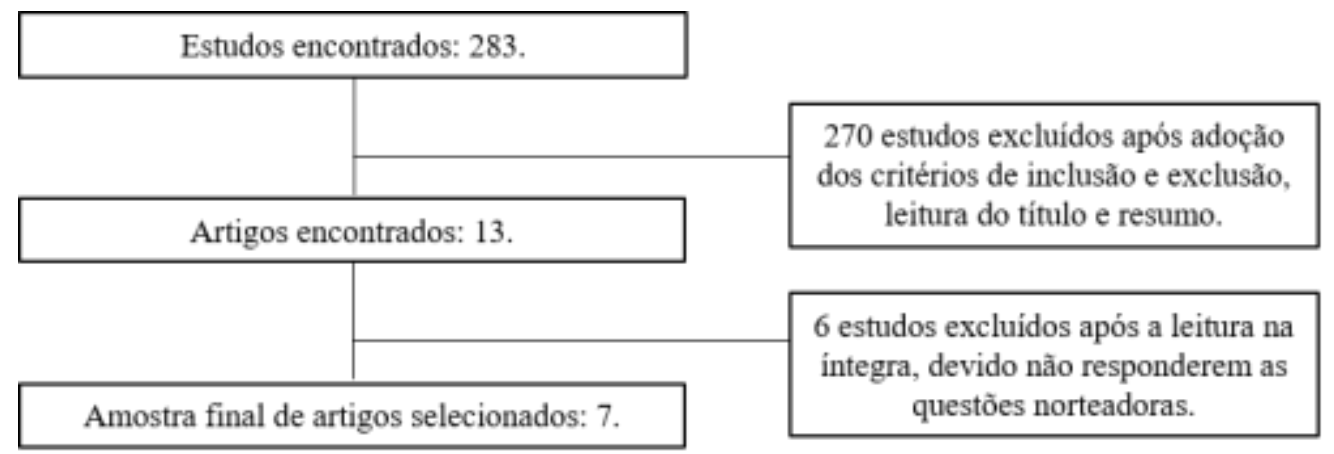

Fonte: Autores.

A partir da extração das informações no formulário de coleta de dados, elaborou-se o Quadro 1, o qual dispõe de informações pertinentes acerca dos artigos selecionados para essa revisão. 
Quadro 1 - Dados dos artigos selecionados na pesquisa. Belém, Pará, Brasil, 2021.

\begin{tabular}{|c|c|c|c|c|c|}
\hline $\mathbf{N}^{\circ}$ & TÍTULO & AUTOR/ANO & REVISTA & OBJETIVOS & MATERIAL E MÉTODO \\
\hline 1 & $\begin{array}{l}\text { Educators' } \\
\text { behaviours } \\
\text { during feedback } \\
\text { in authentic } \\
\text { clinical practice } \\
\text { settings: an } \\
\text { observational } \\
\text { study and } \\
\text { systematic } \\
\text { analysis }\end{array}$ & $\begin{array}{l}\text { Johnson, et al., } \\
2019 .\end{array}$ & $\begin{array}{l}\text { BMC Medical } \\
\text { Education. }\end{array}$ & $\begin{array}{l}\text { O objetivo deste estudo } \\
\text { foi observar e } \\
\text { sistematicamente } \\
\text { comportamento }\end{array}$ & $\begin{array}{l}\text { Educadores e alunos filmaram a si } \\
\text { próprios durante as sessões formais de } \\
\text { feedback no treinamento de rotina do } \\
\text { hospital. Os pesquisadores compararam } \\
\text { a prática dos educadores a um conjunto } \\
\text { publicado de } 25 \text { comportamentos de } \\
\text { educadores recomendados para } \\
\text { feedback de qualidade. Os } \\
\text { comportamentos individuais do } \\
\text { educador foram classificados como } 0= \\
\text { não visto, } 1 \text { = feito de alguma forma, } 2 \\
=\text { feito de forma consistente. Para } \\
\text { caracterizar a prática individual do } \\
\text { educador, seus escores de } \\
\text { comportamento foram somados. Para } \\
\text { descrever a frequência com que cada } \\
\text { comportamento foi observado em todos } \\
\text { os vídeos, as pontuações médias foram } \\
\text { calculadas. }\end{array}$ \\
\hline 2 & $\begin{array}{l}\text { Effects of an } \\
\text { examiner's } \\
\text { positive and } \\
\text { negative } \\
\text { feedback on } \\
\text { self-assessment } \\
\text { of skill } \\
\text { performance, } \\
\text { emotional } \\
\text { response, and } \\
\text { self-efficacy in } \\
\text { Korea: a quasi- } \\
\text { experimental } \\
\text { study. }\end{array}$ & Kim \& Lee, 2019. & $\begin{array}{l}\text { BMC Medical } \\
\text { Education. }\end{array}$ & $\begin{array}{l}\text { Avaliar o esgotamento de } \\
\text { Residentes Médicos. }\end{array}$ & $\begin{array}{l}\text { Este estudo foi um projeto pós-teste } \\
\text { quase experimental para examinar os } \\
\text { efeitos do feedback verbal positivo e } \\
\text { negativo de um examinador sobre a } \\
\text { precisão da autoavaliação, respostas } \\
\text { emocionais e autoeficácia. Estudantes } \\
\text { de enfermagem do segundo ano foram } \\
\text { recrutados em uma universidade na } \\
\text { Coréia do Sul. Um total de } 110 \\
\text { participantes foram atribuídos } \\
\text { aleatoriamente a um grupo de feedback } \\
\text { positivo e um grupo de feedback } \\
\text { negativo. Todos os participantes } \\
\text { completaram a medida de desempenho } \\
\text { e, em seguida, receberam um feedback } \\
\text { positivo ou negativo de um avaliador. } \\
\text { Após a entrega do feedback, eles } \\
\text { avaliaram seu próprio desempenho } \\
\text { usando a mesma folha do avaliador e } \\
\text { completaram a pesquisa para resposta } \\
\text { emocional e autoeficácia. Testes qui- } \\
\text { quadrado, testes exatos de Fisher, } \\
\text { amostra independente de Student. }\end{array}$ \\
\hline 3 & $\begin{array}{l}\text { Análise do } \\
\text { processo } \\
\text { Formativo de } \\
\text { uma Residência } \\
\text { de Enfermagem } \\
\text { em Terapia } \\
\text { Intensiva. }\end{array}$ & Santos, et al., 2017. & $\begin{array}{l}\text { Revista } \\
\text { Baiana de } \\
\text { Enfermagem. }\end{array}$ & $\begin{array}{llr}\text { Analisar } & \text { o } & \text { processo } \\
\text { formativo } & \text { de } & \text { uma } \\
\text { Residência } & & \text { de } \\
\text { Enfermagem em } & \text { Terapia } \\
\text { Intensiva sob a ótica dos } \\
\text { egressos. }\end{array}$ & $\begin{array}{l}\text { Estudo descritivo, exploratório e } \\
\text { quantitativo. Participaram todos os } \\
\text { egressos da residência entre os anos de } \\
2004 \text { a 2012. Deles, } 91 \text { retornaram o } \\
\text { instrumento de pesquisa enviado por e- } \\
\text { mail e preenchido entre julho e } \\
\text { setembro de 2013. Os dados foram } \\
\text { analisados através da estatística } \\
\text { descritiva. }\end{array}$ \\
\hline
\end{tabular}




\begin{tabular}{|c|c|c|c|c|c|}
\hline 4 & $\begin{array}{l}\text { Competency- } \\
\text { based education } \\
\text { in transitioning } \\
\text { nurse } \\
\text { practitioner } \\
\text { students from } \\
\text { education into } \\
\text { practice. }\end{array}$ & $\begin{array}{l}\text { Hodges, et al., } \\
2019 .\end{array}$ & $\begin{array}{l}\text { American } \\
\text { Association of } \\
\text { Nurse } \\
\text { Practitioners. }\end{array}$ & $\begin{array}{l}\text { Este estudo visou discutir } \\
\text { as formas avaliativas para } \\
\text { programas de ensino em } \\
\text { enfermagem, incluindo o } \\
\text { feedback formativo. }\end{array}$ & $\begin{array}{l}\text { Estudo exploratório acerca de como } \\
\text { diferentes modelos de desenvolvimento } \\
\text { em avaliação podem ser implementados } \\
\text { em programas de ensino em } \\
\text { enfermagem. }\end{array}$ \\
\hline 5 & $\begin{array}{l}\text { Experiential } \\
\text { knowledge of } \\
\text { nursing } \\
\text { residence } \\
\text { preceptors: an } \\
\text { ethnographic } \\
\text { study. }\end{array}$ & $\begin{array}{l}\text { Manhães, et al., } \\
2017 .\end{array}$ & $\begin{array}{l}\text { Online } \\
\text { brazilan } \\
\text { jornal } \\
\text { nursing. }\end{array}$ & $\begin{array}{l}\text { Identificar como o } \\
\text { conhecimento } \\
\text { experiencial mobiliza o } \\
\text { saber pedagógico nos } \\
\text { preceptores da residência } \\
\text { médica e discutir } \\
\text { estratégias de formação } \\
\text { de preceptores. }\end{array}$ & $\begin{array}{l}\text { Trata-se de uma pesquisa qualitativa e } \\
\text { etnográfica. Foram realizados } \\
\text { seminário, observação participante e } \\
\text { entrevistas. }\end{array}$ \\
\hline 6 & $\begin{array}{l}\text { Residência em } \\
\text { enfermagem: } \\
\text { identificação } \\
\text { das atividades } \\
\text { de preceptoria } \\
\text { em um Hospital } \\
\text { escola. }\end{array}$ & $\begin{array}{l}\text { Filho \& Santos, } \\
\text { 2018. }\end{array}$ & $\begin{array}{l}\text { O Mundo da } \\
\text { Saúde. }\end{array}$ & $\begin{array}{l}\text { Identificar as atividades } \\
\text { desempenhadas pelos } \\
\text { preceptores da Residência } \\
\text { em Enfermagem do } \\
\text { Hospital das Clínicas em } \\
\text { Pernambuco. }\end{array}$ & $\begin{array}{l}\text { Uma pesquisa de caráter descritivo- } \\
\text { exploratória e quantitativa. Na coleta de } \\
\text { dados utilizou-se um questionário com } \\
16 \text { perguntas baseadas na resolução da } \\
\text { Comissão Nacional de Residência } \\
\text { Multiprofissional em } \\
\text { Saúde; a amostra foi composta por } 36 \\
\text { preceptores em enfermagem. }\end{array}$ \\
\hline 7 & $\begin{array}{l}\text { Elaboração de } \\
\text { projeto } \\
\text { pedagógico para } \\
\text { preceptoria de } \\
\text { enfermeiros em } \\
\text { terapia intensiva } \\
\text { cardiológica. }\end{array}$ & $\begin{array}{ll}\text { Martins } & \& \\
\text { Scarcella, 2020. } & \end{array}$ & $\begin{array}{l}\text { Revista } \\
\text { Nursing. }\end{array}$ & $\begin{array}{l}\text { Relatar a elaboração de } \\
\text { um projeto pedagógico } \\
\text { em preceptoria para } \\
\text { enfermeiros em terapia } \\
\text { intensiva cardiológica. }\end{array}$ & $\begin{array}{l}\text { Estudo descritivo, exploratório, com } \\
\text { abordagem qualitativa, do tipo relato de } \\
\text { experiência, realizado em um Hospital } \\
\text { Universitário Federal. Relato baseado } \\
\text { na experiência de enfermeiras } \\
\text { preceptoras em residência } \\
\text { multiprofissional na área de } \\
\text { Concentração Enfermagem e atuantes } \\
\text { na terapia intensiva cardiológica } \\
\text { durante o ano de 2019. }\end{array}$ \\
\hline
\end{tabular}

Fonte: Autores.

Por meio da análise de conteúdo, proposta por Bardin, foi possível elencar 3 categorias: 1) A importância do Feedback Formativo na formação em residências; 2) O papel do preceptor Enfermeiro perante os residentes e ao Feedback Formativo e 3) Entraves para a atuação na preceptoria em residências e o processo de Feedback Formativo.

\section{A importância do Feedback Formativo na formação em residências}

O feedback é considerado uma das ferramentas mais valiosas no que tange ao processo de ensino-aprendizagem, assim como no desempenho. Haja vista que, oferece a oportunidade para o aluno/residente o benéfico momento de reflexão sobre sua prática assistencial, a partir da crítica construtiva, do raciocínio, do aconselhamento e do apoio de outro profissional - no âmbito das residências, o preceptor. Por meio dessa colaboração, o indivíduo pode aprimorar sua compreensão de quais são as metas de desempenho e como buscar atingir esses padrões (Johnson et al., 2019).

Corroborando Johnson e colaboradores (2019), para Kim e Lee (2019), diante do processo de feedback, os alunos usufruem da possibilidade de obtenção das informações repassadas para a identificação de quaisquer lacunas referentes aos seus desempenhos, para assim buscar melhoraria nas áreas onde apresentam dificuldades, seja ela de desempenho, teórica ou ambas. Para esses autores, o momento de feedback proporciona a capacidade de autoavaliação, sendo esta um dos elementos 
chave para uma aprendizagem autodirigida e baseada no desempenho.

Kim e Lee (2019), classificam o processo de feedback como negativo ou positivo. Nessa perspectiva, os autores pontuam que o feedback negativo consiste em ser utilizado pautando uma atitude e/ou comportamento do aluno/residente ao realizar uma tarefa. Caso a mesma não seja realizada corretamente, o preceptor é incumbido de repassar os pontos em que o aluno não alcançou os níveis esperados, seja a respeito de conhecimentos técnicos ou teóricos para suprir os objetivos esperados. Dessa forma, o feedback negativo indica quando é necessária uma mudança de comportamento. Em contrapartida a isso, o feedback positivo é utilizado para indicar quando o aluno/residente atinge os níveis esperados na execução de alguma tarefa, ou apresenta comportamento que seja esperado diante determinadas situações. Esse tipo de feedback é utilizado para reforçar atitudes eficazes em relação a uma meta.

Dessa forma, compreende-se o feedback como uma ferramenta de extrema importância no processo formativo em saúde, em especial na ambiência das residências. Uma vez que se fazem necessários os direcionamentos repassados pelos preceptores em relação as atividades que cerceiam o processo de ensino-aprendizagem dos residentes no desenvolvimento de habilidades técnicas e teóricas. Assim, tem-se na figura do preceptor o viés que norteia o processo crítico e reflexivo dos residentes, para que os mesmos possam realizar suas autoavaliações e buscarem a manutenção ou a melhora de comportamentos.

\section{O papel do preceptor perante os residentes e ao Feedback Formativo}

Conforme Martins e Scarcella (2020), o residente, durante seu processo formativo, necessita de educação permanente no serviço, com vistas a subsidiar suas práticas e intervenções pautadas em evidências cientificas, bem como conhecimento teórico de qualificado. Dessa forma, a preceptoria surge a fim de possibilitar a resolução desta demanda e o preceptor desempenha uma função de educador no serviço de saúde.

Nessa conjuntura, o profissional preceptor detém importante papel pedagógico no que tange ao aprimoramento no processo ensino-aprendizagem dos residentes. Dessa forma, esses profissionais devem, de acordo com Filho e Santos (2018), fornecer feedback, desenvolver postura pedagógica, compartilhar suas experiências profissionais, deter capacidade de disseminar o ensino e a aprendizagem. Além de aconselhar, influenciar e inspirar, desenvolver e conhecer a capacidade intelectual de cada residente.

A execução da preceptoria, no âmbito dos programas de residência, exige dessa forma, dos preceptores, além de com competências técnicas, competências e habilidades pedagógicas a fim de realizar o escopo dos programas de especialização em serviço, que é a mediação entre a teoria e a prática (Martins \& Scarcella, 2020; Ribeiro et al., 2020)

Manhães e colaboradores (2017) e Melo, Queluci e Gouvea (2014), apontam que se recomenda que os preceptores devem possuir conhecimentos teóricos, didáticos e políticos, além disso, sua vivência se torna viés fundamental para vislumbrar a união e articulação da formação em saúde ao mercado de trabalho. Nesse contexto, esses autores evidenciam que o preceptor não desempenha somente função na formação de competências e técnicas próprias da profissão, mas também influencia acerca da humanização, da ética e na formação de opinião de quem os acompanha.

Em consonância a recomendação relacionada a vivencia, Manhães e seus colaboradores (2017), apontam importante fato, o qual pode ser levado em consideração ao eleger o corpo preceptor. Os pesquisadores constataram que, os preceptores que já haviam participado do programa de residência, na condição de residentes, desenvolveram uma supervisão mais empática dos residentes. Dessa forma, entende-se que essa experiência pregressa demonstra que pode auxiliar o preceptor no desenvolvimento de sua prática pedagógica, uma vez que se assemelha à experiência que os residentes estão vivenciando. Haja vista que, os preceptores que, outrora, foram residentes de enfermagem traziam consigo uma sensibilidade para a prática da tutoria que contribui para a compreensão das demandas de aprendizagem dos residentes, o que os capacitou a utilizarem 
melhores formas de propulsionar o ensino-aprendizagem.

Corroborando isso Hodges et al. (2019), evidenciou que processo de feedback formativo partindo do preceptor clínico é de suma importância para colaborar na elevação da competência clínica e a prontidão para a prática. Visto que os preceptores clínicos podem passar muito tempo supervisionando os alunos/residentes, conferindo-lhes uma experiência de ensinoaprendizagem de forma mais imersiva, ao correlacionar achados teóricos com o cotidiano assistencial.

No entanto, percebe-se que, muitos profissionais que desempenham atividades de preceptoria nos serviços de saúde, não dispõem dessa experiência pregressa. Tais fatores acabam por acarretar em dificuldades ao desempenharem seus papeis, não correspondendo às expectativas em algumas particularidades, como a realização de feedbacks.

\section{Entraves para a atuação na preceptoria em residências e o processo de Feedback Formativo}

Pesquisa de Johnson et al. (2019), evidenciou uma das problemáticas mais recorrentes no que tange ao processo de feedback formativo, indicando que, ainda que os preceptores façam comentários sobre aspectos específicos do desempenho, ensine e/ou relembrem conceitos importantes e descrevem como o residente pode melhorar, os preceptores tendem a ter o domínio da fala na maior parte do tempo durante as rodadas de feedbacks, inviabilizando e dificultando o processo de autoavaliação, bem como a criação de um plano de ação de objetive a melhora em pontos que sejam pertinentes.

Amparada nesta problemática, Schumacher e Risco (2017) ratificados por Hodges et al. (2019), pontuam que, por parte dos preceptores, a falta de uma linguagem e método comuns para tomar decisões sobre o desempenho dos residentes/alunos dificultam a progressão das seções de feedback formativo e, por consequência, a avaliação das habilidades.

Ainda conforme Johnson e seus colaboradores (2019), outro viés problemático que caracterizou o processo de feedback formativo foi a ausência da criação de um ambiente de aprendizagem seguro no início da sessão, por parte dos educadores, sendo este um empecilho para a eficácia objetivada, embora eles mostrassem respeito e apoio pelos alunos em geral. Para esses autores, é de extrema importância a promoção de um ambiente de aprendizagem seguro, partindo do ponto de vista que esta pode ser considerada uma estratégia perspicaz quando o preceptor e o residente não possuem uma relação estabelecida, o que parece ser cada vez mais comum devido a rotatividade dos residentes entre os setores.

Em pesquisa realizada por Santos e seus colaboradores (2017), com egressos de programas de residência em enfermagem em Unidade de Terapia Intensiva, evidenciou-se insatisfações no relacionamento interpessoal entre os residentes com o corpo preceptor. Sendo este fator diretamente ligados à condução do processo de ensino-aprendizagem dos residentes. Destaca-se que a manutenção de um bom relacionamento interpessoal com todos os envolvidos no processo de treinamento é de suma importância para manter a harmonia nos ambientes de trabalho. Logo, entende-se que a relação com a preceptoria é um indicativo que repercute na qualidade do aprendizado proporcionado pela residência.

Segundo Manhães e colaboradores (2017), é fundamental que os preceptores tenham uma "disposição pedagógica" associada à sua prática com os residentes. No entanto, observa-se que, poucos preceptores detêm dessas habilidades de cunho pedagógico para mediar os processos que cerceiam a preceptoria. Dessa maneira, entende-se que esse entrave pode ser uma possível justificativa que acarreta nas problemáticas já evidenciadas.

Filho e Santos (2018), indicam que, grande parte dos preceptores detêm domínio sobre profissional no que diz respeito às suas áreas de atuações. No entanto, esses profissionais não apresentam a disposição pedagógica frisada por Manhães e seus colaboradores (2017). Sendo assim, cabe às instituições o papel de preparar essa formação pedagógica do o conteúdo preceptor.

Observa-se que há pouca participação por parte dos profissionais na implementação do Projeto Pedagógico do programa da Residência. Isso contribui para que os preceptores não conheçam a fundo suas atribuições, logo quando pouco se se conhece, pouco se realiza suas atribuições de forma adequada, não se podendo realizar suas atividades de forma plena e que 
atendam as reais demandas dos residentes, como seções de feedback formativo eficazes (Filho \& Santos, 2018).

Tais fatos se confirmam pelos relatos dos participantes da pesquisa de Manhães, onde uma das preceptoras enfermeiras relata que não estava preparada para desempenhar tal função: "Na verdade, não estava preparado para ser um preceptor. Eu estava preparada para ser enfermeira. Então, nossa prática é mostrada a eles, mas não temos uma rotina formal ou algo parecido com eles (residentes) [...]” (Manhães et al. 2017).

Aliado a isso, Manhães et al. (2017), retrata ainda que na dificuldade de reconhecer seu papel educativo, os preceptores acabam por enxergar os residentes como uma carga extra de trabalho, repercutindo na sobrecarrega de tarefas no cotidiano assistencial dos enfermeiros. Esses autores ainda evidenciam que alguns enfermeiros não conseguem articular efetivamente a prática de atividades assistenciais com as de preceptor. Nessa perspectiva, entende-se que esse fator reverbera consideravelmente no que tange aos processos de feedback formativo, além de colaborar para a efetivação e perpetuação de outras problemáticas já evidenciadas, como a questão do relacionamento interpessoal.

\section{Considerações Finais}

A partir dessa revisão de literatura, foi possível identificar a importância do feedback formativo, bem como da preceptoria na ambiência das residências de enfermagem. Além disso, identificaram-se os principais entraves para a implementação e exercício da preceptoria e feedback, como a falta de preparo pedagógico dos profissionais e as dificuldades no reconhecimento do seu papel.

Assim, é inegável a relevância do feedback formativo com vistas a propulsionar o pensamento crítico e reflexivo do residente durante sua formação de especialização. No entanto, esse processo depende diretamente de um preceptor que reconheça e realize sua função de forma satisfatória, o que, muitas vezes, acaba por não ser a realidade devido à falta de incentivos e amparo institucional para o fomento de tais práticas.

Como limitações desse estudo, elenca-se a escassez dessa temática na literatura, onde pouco pôde-se correlacionar o feedback e a preceptoria na ambiência das residências de enfermagem. Dessa forma, sugere-se que mais estudos específicos sejam realizados nessa área temática, com vistas a fomentar e disseminar o debate acerca desse importante assunto dada a relevância do feedback formativo e visto que os preceptores desempenham importante papel na formação dos residentes.

\section{Referências}

Bardin, L. (2016). Análise de Conteúdo. Revista Eletrônica de Educação, São Paulo, ed. 70, p. 229.

Borges, M. C., Miranda, C. H., Santana, R. C., \& Bollela, V. R. (2014). Avaliação formativa e feedback como ferramenta de aprendizado na formação de profissionais da saúde. Medicina (Ribeirão Preto), 47(3), 324-331. https://doi.org/10.11606/issn.2176-7262.v47i3p324-331

Brasil (1996). Conselho Federal de Enfermagem. Anteprojeto de Lei no 2.264/1996. Dispõe sobre a Residência em Enfermagem e a sua respectiva Comissão Nacional. Rio de Janeiro. https://www.camara.leg.br/proposicoesWeb/fichadetramitacao?idProposicao=17872

Brasil (2014). Ministério da Educação. Resolução n ${ }^{\circ}$, de 7 de novembro de 2014. Dispõe sobre a duração e a carga horária dos programas de Residência em Área Profissional da Saúde nas modalidades multiprofissional e uniprofissional e sobre a avaliação e a frequência dos profissionais da saúde residentes. Diário Oficial União. https://www.legisweb.com.br/legislacao/?id=276672

Fajardo, A. P., Rocha, C. M. F., \& Pasini, V. L. (2010). Grupo Hospitalar Conceição. Residências em saúde: fazeres e saberes na formação em saúde. Porto Alegre: Hospital Nossa Senhora da Conceição.

Ferreira, F. D. C., Dantas, F. C., \& Valente, G. S. C. (2018). Nurses' knowledge and competencies for preceptorship in the basic health unit. Rev Bras Enferm, 71(Suppl 4):1564-1571. http://dx.doi.org/10.1590/0034-7167-2016-0533

Filho, J. F. R., \& Santos, C. S. (2018). Residência em enfermagem: identificação das atividades de preceptoria em um Hospital escola. $O$ Mundo da Saúde, 42(2), 333-348. https://revistamundodasaude.emnuvens.com.br/mundodasaude/article/view/127

Freitas, B. T. P., Sé, A. C. S., da Silva Gonçalves, R. C., \& Pereira, G. L. (2021). Contribuições e desafios da preceptoria nos Programas de Residência em Enfermagem. Research, Society and Development, 10(5), e37510514996-e37510514996. https://doi.org/10.33448/rsd-v10i5.14996 
Research, Society and Development, v. 10, n. 14, e397101419849, 2021 (CC BY 4.0) | ISSN 2525-3409 | DOI: http://dx.doi.org/10.33448/rsd-v10i14.19849

Hodges, A. L., Konicki, A. J., Talley, M. H., Bordelon, C. J., Holland, A. C., \& Galin, F. S. (2019). Competency-based education in transitioning nurse practitioner students from education into practice. Journal of the American Association of Nurse Practitioners, 31(11), 675-682. https:// doi:10.1097/JXX.0000000000000327

Johnson, C. E., Keating, J. L., Farlie, M. K., Kent, F., Leech, M., \& Molloy, E. K. (2019). Educators' behaviours during feedback in authentic clinical practice settings: an observational study and systematic analysis. BMC medical education, 19(1), 1-11. https://doi.org/10.1186/s12909-019-1524-z

Kim, E. J., \& Lee, K. R. (2019). Effects of an examiner's positive and negative feedback on self-assessment of skill performance, emotional response, and self-efficacy in Korea: a quasi-experimental study. BMC medical education, 19(1), 1-7. https://doi.org/10.1186/s12909-019-1595-x

Manhães, L. S. P., de Melo Tavares, C. M., Ferreira, R. E., Marcondes, F. L., da Silveira, P. G., \& Lima, T. O. (2017). Experiential knowledge of nursing residence preceptors: an ethnographic study. Online Brazilian Journal of Nursing, 16(3), 277-288. https://doi.org/10.17665/1676-4285.20175464

Martins, G. M., Caregnato, R. C. A., Barroso V. L. M., \& Ribas, D. C. P. (2016). Implementação de residência multiprofissional em saúde de uma universidade federal: trajetória histórica. Rev Gaúcha Enferm, 37(3):e57046. http://dx.doi.org/10.1590/1983-1447.2016.03.57046.

Martins, L. S., \& Scarcella, M. F. S. (2020). Elaboração de projeto pedagógico para preceptoria de enfermeiros em terapia intensiva cardiológica. Nursing (São Paulo), 23(269), 4695-4702. https://doi.org/10.36489/nursing.2020v23i269p4695-4702

Melo, M. C. D., Queluci, G. D. C., \& Gouvêa, M. V. (2014). Problematizando a residência multiprofissional em oncologia: protocolo de ensino prático na perspectiva de residentes de enfermagem. Revista da Escola de Enfermagem da USP, 48(4), 706-714. https://doi.org/10.1590/S0080-623420140000400019

Ribeiro, P. K. C., Firmo, W. C. A., Sousa, M. H. S. L., Figueiredo, I. A., \& Pacheco, M. A. B. (2020). Os profissionais de saúde e a prática de preceptoria na atenção básica: assistência, formação e transformações possíveis. Journal of Management \& Primary Health Care, 12, 1-18. https://doi.org/10.14295/jmphc.v12.977

Santos, A. D. S., Castro, L. M. C., Fagundes, N. C., \& Vieira, D. F. V. B. (2017). Análise do processo formativo de uma residência de enfermagem em terapia intensiva. Revista Baiana de Enfermagem31,(4). https://cienciasmedicasbiologicas.ufba.br/index.php/enfermagem/article/viewFile/22771/15399

Schumacher, G., \& Risco, K. (2017). Competency-based nurse practitioner education: an overview for the preceptor. The Journal for Nurse Practitioners, 13(9), 596-602. https://doi.org/10.1016/j.nurpra.2017.07.020

Sousa, L. M. M., Marques-Vieira, C. M. A., Severino, S. S. P., \& Antunes, A. V. (2017). A metodologia de revisão integrativa da literatura em enfermagem. Revista investigação em enfermagem, https://www.researchgate.net/publication/321319742_Metodologia_de_Revisao_Integrativa_da_Literatura_em_Enfermagem

17-26

